\title{
Finite Element Simulation of the Thermo- mechanical Response of Graphene Reinforced Nanocomposites
}

\author{
Androniki S. Tsiamaki, and Nick K. Anifantis ${ }^{1}$ \\ Department of Mechanical Engineering and Aeronautics, University of Patras , 26504 Patras, Greece
}

\begin{abstract}
The research for new materials that can withstand extreme temperatures and present good mechanical behavior is of great importance. The interest is highly focused on the utilization of composites reinforced by nanomaterials. To cope with this goal the present work studies the mechanical response of graphene reinforced nanocomposite structures subjected to temperature changes. A computational finite element model has been developed that accounts for both the reinforcement and the matrix material phases. The model developed is based on both the continuum theory and the molecular mechanics theory, for the simulation of the three different material phases of the composite, respectively, i.e. the matrix, the intermediate transition phase and the reinforcement. Considering this model, the mechanical response of an appropriate representative volume element of the nanocomposite is simulated under various temperature changes. The study involves different types of reinforcement composed from either monolayer or multilayer graphene sheets. Apart from the investigation of the behavior of a nanocomposite with each particular type of the reinforcement, comparisons are also presented between them in order to reveal optimized material combinations. The principal parameters taken into consideration, which contribute also to the mechanical behavior of the nanocomposite, are its size, the sheet multiplicity as well as the volume fraction.
\end{abstract}

\section{Introduction}

Since their discovery, nanomaterials have occupied a lot the scientific community due to their excellent mechanical, thermal and electrical properties. Those materials enable new extraordinary applications and allow the realization of new products with enhanced properties than the conventional ones. Also, the need for materials that exhibit great mechanical properties even in extreme temperatures is increasing. Nanomaterials can as well fulfill this requirement. The most amazing among them are carbon allotropes such as graphene, a two dimensional hexagonal lattice of one-atom thickness composed of carbon atoms, and carbon nanotubes (CNTS), tubes composed of rolled-up graphene. Especially, graphene's mechanical properties have been extensively studied both in theoretical [1-6]

${ }^{1}$ Corresponding author: nanif@upatras.gr 
and experimental [7-9] works. There are also some studies, mainly theoretical ones $[5,10,11]$, that deal with the thermomechanical properties of graphene on various temperatures.

Due to its great properties, graphene is a candidate material for reinforcing other common materials such as ceramics and polymers. Polymeric matrices reinforced with graphene have been widely studied from several points of view, either for mechanical or electrical or thermal properties. It has been found that the mechanical properties of polymeric materials can be highly enhanced [12,13], approximately $16 \%$, when reinforced by graphene. Apart from excellent mechanical behavior, graphene nanocomposites exhibit great thermal properties $[14,15]$ such as thermal conductivity and coefficient of thermal expansion. Nevertheless, having monolayer graphene sheets ideally contributed on the matrix does not always happen as it is possible to not have uniform dispersion of the graphene or even have agglomerations. Hence, graphene can be met in multilayer stacks, which has been found to be less stiff than single layer. So, it would be very interesting to investigate the mechanical behavior of a polymer reinforced by either monolayer or multilayer graphene and compare the difference, if any, between different types of reinforcement. In addition to this, due to the requirement of new materials that can perform well in extreme environmental temperatures, is of great importance to study how such a nanocomposite would behave on temperature change.

This paper aims to investigate the thermomechanical behavior of polymer nanocomposites reinforced with single layer or multilayer graphene sheets using a multiscale modeling analysis. This work can be separated on two stages of analysis. On the first one, graphene is modeled using the atomistic approach based on the molecular mechanics theory. In this approach, the interatomic interactions are simulated as springs of specific stiffness that change due to temperature and so, the mechanical properties of graphene on various temperatures are obtained. On the second stage of the proposed analysis, we use the graphene properties predicted on the first stage in order to build the model of the nanocomposite with graphene as reinforcement. For the simulation of the graphene-based nanocomposite we use a representative volume element (RVE) which includes all three material phases i.e. the reinforcement in the middle, matrix on both sides of it and the intermedium phase between them. The parameters considered are the number of graphene layers and its volume fraction on the nanocomposite. Finally, the mechanical dependent properties of the composite are calculated based on the classical elastic theory using appropriate boundary conditions.

\section{Methodology}

\subsection{Multiscale modeling analysis}

\subsubsection{Modeling the thermomechanical behavior of graphene}

Beginning the analysis on the first stage, graphene is modeled based on molecular mechanics theory according to its atomistic structure. Spring elements have been used for the simulation of the interatomic interactions and nodes at the exact positions of the carbon atoms in the hexagonal lattice. Also, the temperature effect is incorporated on the model in order to predict its thermomechanical response. As known, when heat is added to a material, its atoms vibrate faster and the space they occupy increases. So, the temperature affects the atomic bond by changing its length i.e. the distance between two neighbor atoms. Thus, we use the following relationship between the bond distance and 
environmental temperature in order to introduce the effect of environmental temperature on interatomic interactions' force constants [16]:

$$
r^{T}{ }_{c c}=r_{c c}^{0}(1+\alpha \Delta T)
$$

where $r_{c c}^{0}=0.1421 \mathrm{~nm}$ is the carbon-carbon bond distance at room temperature, $\alpha$ is coefficient of thermal expansion of carbon-carbon bond given by Chen et al. [17] and $\Delta T$ is the temperature variation. Considering the bonding as linear while the bond distance represents the average equilibrium length at a particular temperature, we find the mechanical properties of graphene as a fucntion of temperature by particularly changing the carbon-carbon bond length.

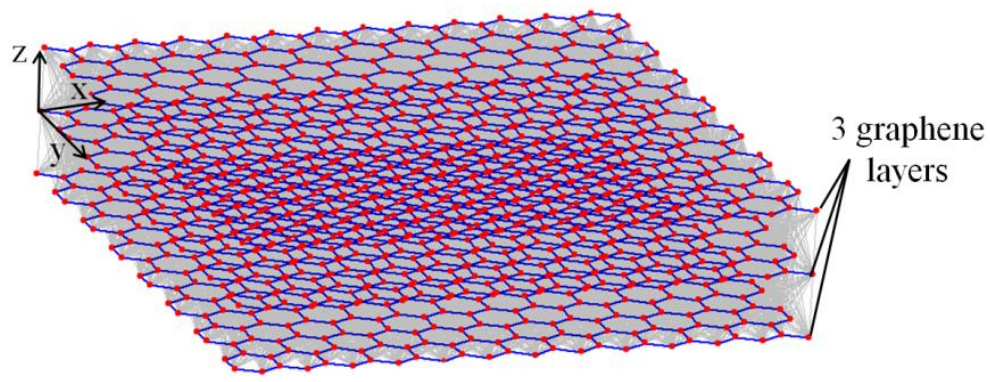

Fig. 1. Representation of a triple layer graphene model.

Moreover, apart from monolayer graphene, we also simulated multilayer graphene sheets where, apart from interatomic interactions, also the van der Waals (vdW) interactions have been incorporated on the model. Figure 1 depicts a graphene sheet of three layers model with vdW bonds that are simulated as spring elements of stiffness depending on the distance between the carbon atoms belonging on two different sheets. The distance between two graphene sheets is equal to monolayer graphene thickness, $t^{g}=0.34 \mathrm{~nm}$. The distance between carbon atoms so that a $\mathrm{vdW}$ bond exists must be maximum $0.85 \mathrm{~nm}$. It should be mentioned that $\mathrm{vdW}$ interactions are weaker than the covalent bonds on the plane of a graphene sheet something that gets this structure less stiff.

\subsubsection{Representative volume element of graphene reinforced polymer}

The thermomechanical properties of graphene, obtained on the first stage of analysis, are used for the simulation of graphene as continuum medium on the second stage of the multiscale modeling proposed. For the analysis of graphene reinforced composite, a representative volume element (RVE) has been modeled and used as shown in Figure 2. This volume element consists of the three parts of the nanocomposite i.e. the matrix, which here has been chosen Poly methyl-methacrylate (PMMA), the reinforcement (either monolayer or multilayer) and the interphase. All three parts of the nanocomposite have been modeled as continuum media with different mechanical properties using three dimensional (3D) solid elements.

The PMMA matrix Young's modulus is $E^{m}=2.5 \mathrm{GPa}$ and its Poisson ratio is $v^{m}=0.34$ at room temperature $T=300 \mathrm{~K}$. For temperatures other than room temperature, PMMA mechanical properties are calculated according to [18]. The thermo-mechanical properties of graphene sheets obtained are shown in Figure 4 that illustrates Young's modulus for monolayer, double-layer and triple-layer. The interphase mechanical properties are determined and bounded by the two materials on its both sides. For the mechanical properties variation of the interphase we have used a linear approximation expressed by an equation of the form $\mathrm{M}=\mathrm{az}+\mathrm{b}$. In this equation, $\mathrm{M}$ stands for the mechanical property of the 
interphase, $\mathrm{z}$ is the axis of materials' thickness that is also axis of the mechanical properties' variation. At the contact points, $M$ equals the mechanical properties of graphene or PMMA respectively. The terms $\mathrm{a}, \mathrm{b}$ are calculated solving the above linear expression for the two bounding situations.

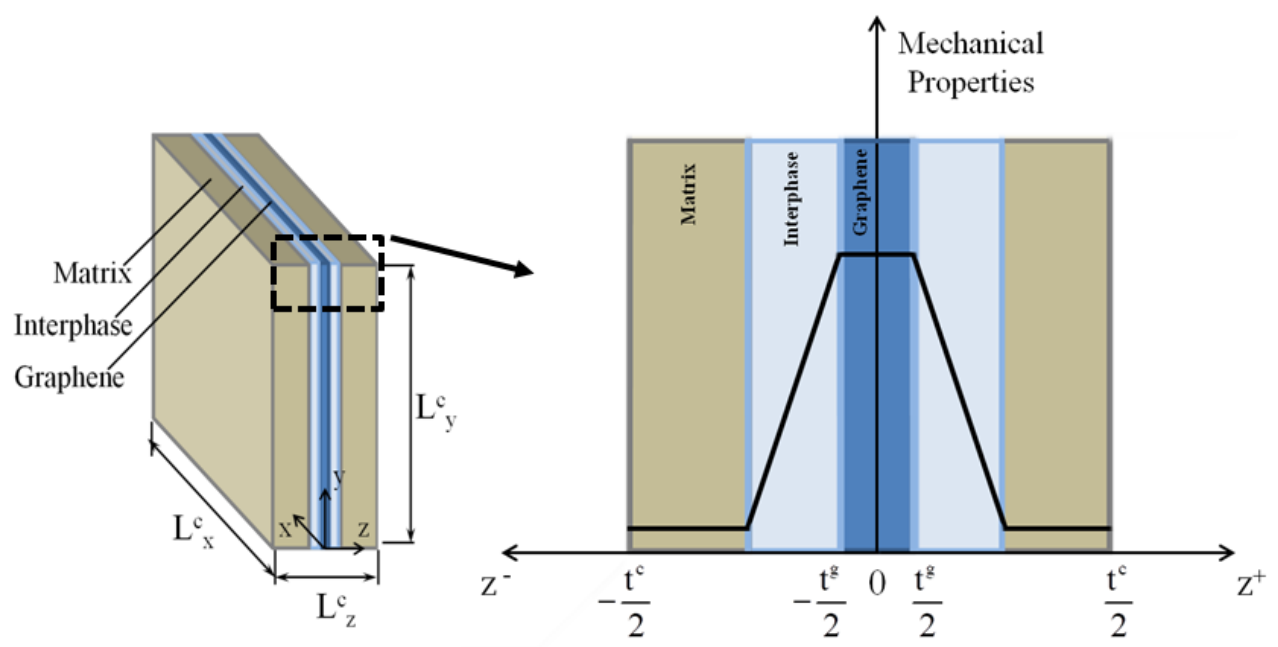

Fig. 2. Schematic view of the volume element of graphene reinforced nanocomposite (left side) and Variation of the mechanical properties of the interphase between two materials (right side).

The interface thickness is of great importance for the prediction of nanocomposite mechanical properties. There have been proposed several values for it varying from $0 \mathrm{~nm}$ to $30 \mathrm{~nm}$. In this study, the interphase thickness is taken equal to $\mathrm{t}^{\mathrm{g}} / 2$ [19]. Due to the fact that interphase, has been connected to phenomena affecting the overall behavior of nanocomposites and in order to linearly and smoothly approximate the variation between the two materials, we have discretised the interphase in eight equally thick parts (Figure 3) along $\mathrm{z}$ axis on both sides so as to model the variation of the interphase mechanical properties. So, the interphase material properties vary in a piecewise manner approaching the linear variations considered.

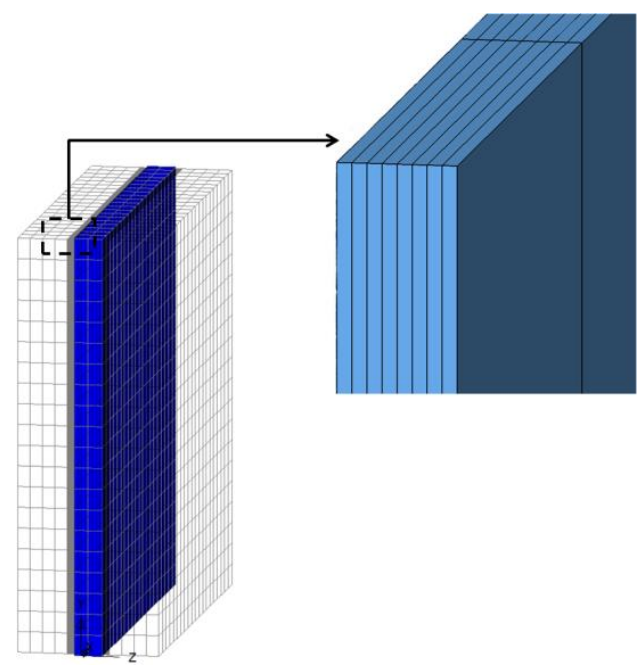

Fig. 3. 3D mesh of graphene reinforced nanocomposite model and magnified mesh view of the interphase. 
Graphene has been reported to behave great on extremely high temperatures that reach even 2000K [20-22]. On the other hand, PMMA cannot. So, we are going to investigate a narrow temperature range from $0 \mathrm{~K}$ to $600 \mathrm{~K}$ that PMMA can barely withstand. For the temperatures examined, we know the mechanical properties of two components (graphene and PMMA). Therefore, the temperature-dependent mechanical properties of the interphase are calculated at each temperature using the linear approximation described.

The considered RVE has constant dimensions $L^{c}{ }_{x}=L^{c}{ }_{y}=10 \mathrm{~nm}$ that are equal to those of graphene sheet. The parameters taken into consideration are the temperature, the number of graphene layers and the volume fraction given by the following equation:

$$
V_{f}=V^{g} / V^{c}=t^{g} / t^{c}=0.34 n / t^{c}
$$

where $V^{g}$ is graphene volume, $V^{c}$ is the RVE volume, $t^{g}$ is graphene thickness, $t^{c}$ is the RVE thickness and $n$ is the number of graphene layers. The volume fractions studied are up to $25 \%$. The volume fraction upper limit is determined by Eq. 2 and especially by the reinforcement thickness. It is obvious that, as that thickness of reinforcement increases i.e. more graphene layers, higher volume fractions are permitted.

While graphene presents orthotropic behavior in armchair and zigzag directions, this difference is low and hence, we consider graphene as isotropic material in our analysis with mechanical properties equal to that of the armchair direction $\left(E^{g}{ }_{x}\right)$. In order to calculate the nanocomposite's Young's modulus, one side of it was clamped while a tension loading was applied on the other side and we used the classical elasticity law:

$$
E^{c}{ }_{x}=\sigma / \varepsilon=\left(F / L^{c}{ }_{x} L^{c} y\right) /\left(d L^{c}{ }_{x} / L^{c}{ }_{x}\right)
$$

where $F$ is the tension loading and $d L^{c}{ }_{x}$ is the elongation provoked.

\section{Results and Discussion}

In Figure 4 the monolayer and multilayer graphene sheet Young's modulus versus temperature is depicted. As shown, there is a small variation of Young's modulus, i.e. a decrease until $100 \mathrm{~K}$ and then an increase of the Young's modulus.

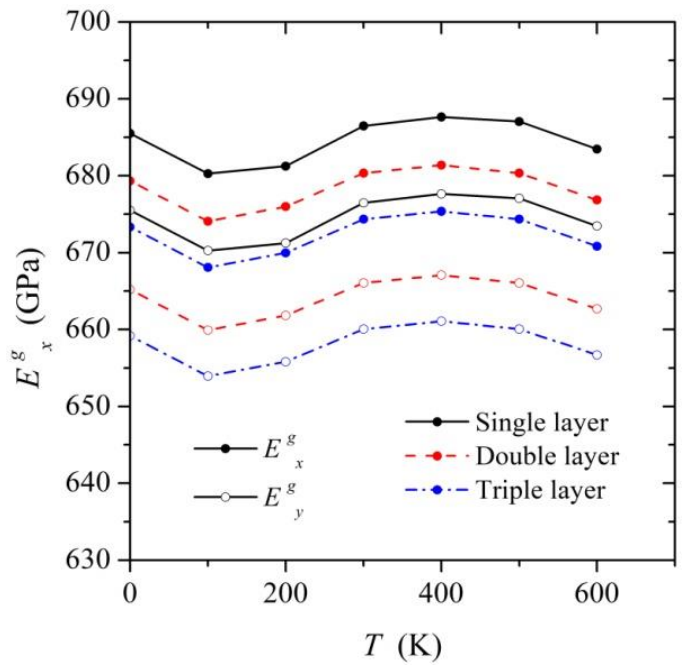

Fig. 4. Graphene Young's modulus variation due to temperature for monolayer and multilayer sheets. 
On the contrary, for multilayer graphene, Young's modulus decreases slightly after 400K. Monolayer graphene is stiffer than multilayer one while there is approximately $10 \mathrm{GPa}$ difference between the Young's modulus in each direction of each type of sheet.

Table 1 contains some comparisons of graphene's mechanical properties at room temperature between the present method and studies from the literature. There is good agreement between the present predictions and studies $[1,2,4,6]$ for Young's modulus. Also, our estimations are close to the values measured in experimental study [7].

Table 1. Comparison of graphene Young's modulus mechanical properties between various methods.

\begin{tabular}{cccc}
\hline Method & Number of layers & $\mathbf{E}^{\mathrm{g}} \mathbf{( T P a )}$ & $\mathbf{v}$ \\
\hline Finite element method [1] & 2 & 0.8 & - \\
\hline Finite element method [2] & 2 & 0.891 & 0.872 \\
\hline \multirow{2}{*}{ Molecular mechanics [3] } & 1 & 1.025 & - \\
\cline { 2 - 3 } Molecular mechanics [4] & 2 & 1.03 & - \\
\hline Molecular Dynamics [5] & 1 & 0.735 & 0.52 \\
\hline Molecular Dynamics [6] & 1 & 0.72 & 0.416 \\
\hline Experimental [7] & 2 & 0.7 & - \\
\hline Experimental [8] & 2 & 0.7 & - \\
\hline & $<5$ & 0.5 & - \\
\hline Present method & 1 & 0.6865 & 0.6076 \\
\hline & 2 & 0.6803 & 0.5965 \\
\hline
\end{tabular}

The results presented deal with the dependence of the nanocomposite Young's modulus due to parameters such as the number of graphene layers, the reinforcement volume fraction and mainly the temperature. In Figure 5, the influence of the temperature on nanocomposite's Young's modulus for various volume fractions is depicted. As shown, the increase of the environmental temperature leads to decrease of Young's modulus.

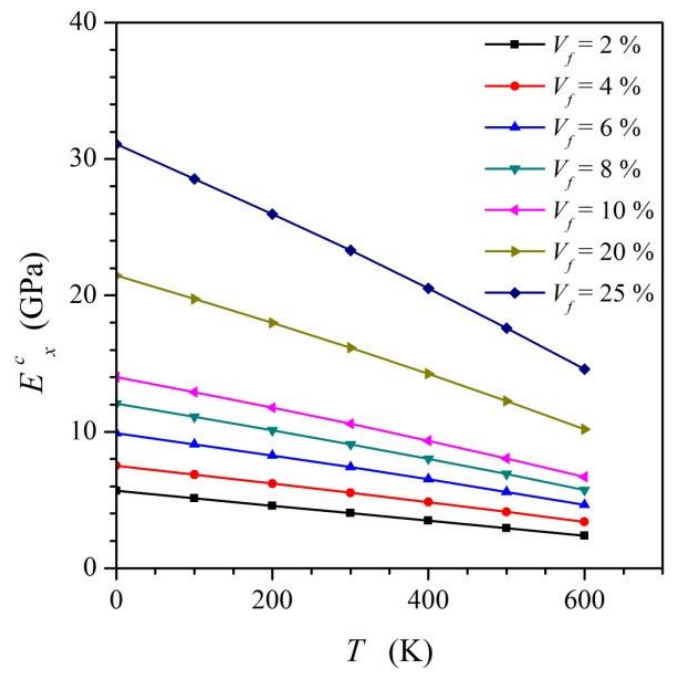

Fig. 5. Influence of composite Young's modulus due to temperature for various monolayer graphene volume fractions.

This decrease tends to have higher inclination for higher volume fractions where we observe better mechanical response of the composite. Also, as expected when the 
reinforcement volume fraction increases, the PMMA becomes stiffer even for high temperatures. However, although graphene surely improves PMMA composite Young's modulus, its dependence on the temperature does not have the same tendency as that of graphene itself (Figure 4).

Another important parameter that must be investigated is the number of graphene layers in the reinforcement due to the fact that, except monolayer graphene, more layers tend to make the structure more unstable. In Figure 6, the variation of the nanocomposite Young's modulus as a function of the temperature is presented for various numbers of graphene layers. Increase of temperature results in lower nanocomposite Young's modulus. It is evident that the lower the graphene sheets' number, the higher the nanocomposite Young's modulus. Also, the nanocomposite with single layer graphene presents approximately two times higher stiffness than that reinforced with the double layer. Apart from this high difference between composite reinforced with monolayer or double layer graphene, as the number of the graphene layers gets higher, there is not much difference. More specifically, over three layers the nanocomposite does not present that significant difference with its previous one containing one layer less.

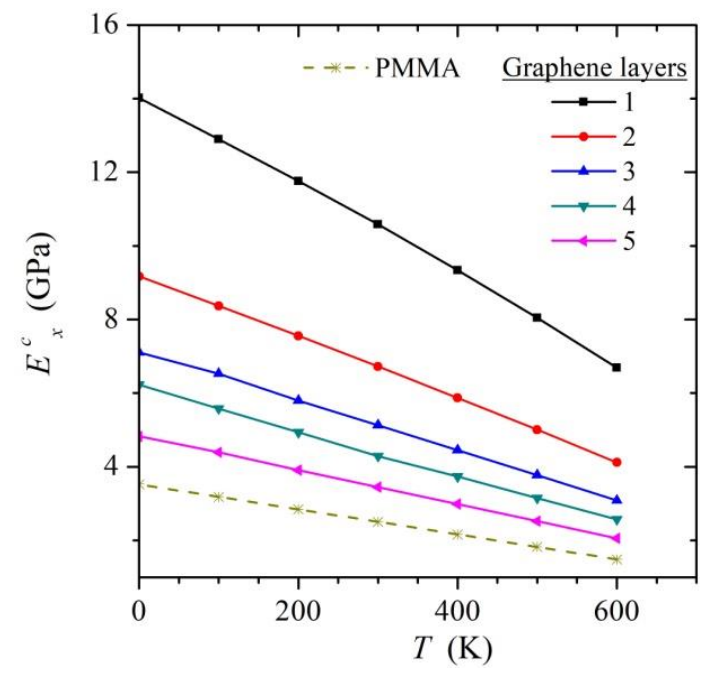

Fig. 6. Influence of composite Young's modulus due temperature for different number of graphene layers.

\section{Conclusions}

This study investigates the thermomechanical behavior of graphene reinforced PMMA nanocomposites. The parameters taken into consideration are the volume fraction and the number of graphene sheets used as reinforcement. Apart from the investigation of the behavior of a nanocomposite with each particular type of reinforcement, comparisons are also presented between them in order to reveal the differences due to reinforcement material. Although the increase of the environmental temperature leads to decrease of Young's modulus, graphene based polymer nanocomposites exhibit high stiffness even in high temperatures. Also, when the reinforcement volume fraction increases, the PMMA becomes stiffer (approximately 9\% of stiffness enhancement) for all temperatures. Moreover, the number of graphene sheets reinforcement affects the nanocomposite Young's modulus. More specifically, the lower the graphene sheets' number, the higher the nanocomposite Young's modulus with highest of all that of the nanocomposite reinforced 
with monolayer graphene. In particular, the nanocomposite with single layer graphene has approximately double stiffness than that with the double layer. As the number of the layers gets higher, the nanocomposite stiffness is not significantly affected. To conclude, the highest PMMA nanocomposite stiffness can be achieved by using monolayer graphene in high volume fractions.

\section{References}

1. M. Neek-Amal, F.M. Peeters, Phys. Rev. B 81, 23 (2010)

2. N. Khandoker, S. Islam, Y.S. Hiung, IOP Conf. Ser.: Mater. Sci. Eng. 206 (2017)

3. C. Li, T.-W. Chou, Composites Science and Technology 63, 1517-1524, (2003)

4. Z. Xu, Computational and Theoretical Nanoscience 6, 1-3 (2009)

5. S.K. Georgantzinos, G.I. Giannopoulos, N.K. Anifantis, J. Appl. Phys., 120 (2016)

6. S. Debroy, V.P. Kumar, K.V. Sekhar, S.G. Acharyya, A. Acharyya, Superlattices Microstruct. 110, 205-214 (2017)

7. R. Rasuli, A. Iraji Zad, M.M. Ahadian, Nanotechnology 21 (2010)

8. I.W. Frank, D.M. Tanenbaum, A.M. van der Zande, P.L. McEuen, J. Vac. Sci. Technol. B 25, 2558-2561 (2007)

9. O.L. Blakslee, D.G. Proctor, E.J. Seldin, G.B. Spence, T. Weng, J. Appl. Phys., 41, 3373-82 (1970)

10. L. Bian, H. Zhao, Compos. Struct. 121, 337 (2015)

11. S. Thomas, M.S. Mrudul, K.M. Ajith, M.C. Valsakumar, J. Phys.: Conf. Ser., 759, 012048 (2016)

12. G.I. Giannopoulos, I.G. Kallivokas, Finite Elem. Anal. Des. 90, 31-40 (2014)

13. J. Cho, J. Luo, I. Daniel, Compos. Sci. Technol., 67 2399-2407, (2007)

14. S. Vadukumpully, J. Paul, N. Mahanta, S. Valiyaveettil, Carbon 49, 198-205 (2011)

15. Y.-H. Zhao, Y.-F. Zhang, S.-L. Bai, X.-W. Yuan, Composites Part B 94, 102-108 (2016)

16. A.K. Rappe, C.J. Casewit, K.S. Colwell, W.A. Goddard III, W.M. Skiff, J. Am. Chem. Soc., 114, 10024-10039 (1992)

17. X. Chen, X. Wang, and B. Y. Liu, J. Reinf. Plast. Compos. 28, 551 (2009)

18. H.-S. Shen, Y. Xiang, F. Lin, D. Hui, Composites Part B 119, 67-78 (2017)

19. S.K. Georgantzinos, G.I. Giannopoulos, N.K. Anifantis, Theor. Appl. Fract. Mech. 52, 158-164 (2009)

20. J.-W. Jiang, J.-S. Wang, and B. Li, Phys. Rev. B 80, 205429 (2009)

21. S. Q. Zhu and X. Wang, J. Therm. Stress. 30, 1195 (2007)

22. N. Mounet, N. Marzari, Phys. Rev. B 71, 205214 (2005) 